\section{BRAZIULIAN JOURNAL \\ OF MEDICAL AND BIOLOGICAL RLSF.ARCH}

www.bjournal.com.br
ISSN 0100-879X

Volume 42 (10) 870-992 October 2009

BIOMEDICAL SCIENCES

AND

CLINICAL INVESTIGATION

Braz J Med Biol Res, October 2009, Volume 42(10) 882-891

High expression of the circadian gene mPer2 diminishes the radiosensitivity of $\mathrm{NIH} 3 \mathrm{~T} 3$ cells

L. Chang, Y.Y. Liu, B. Zhu, Y. Li, H. Hua, Y.H. Wang, J. Zhang, Z. Jiang and Z.R. Wang

The Brazilian Journal of Medical and Biological Research is partially financed by
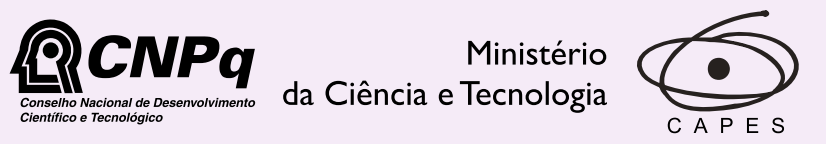

Ministério da Educação

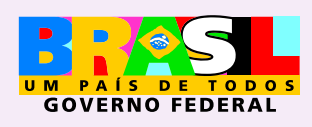

DFAPESP

Institutional Sponsors 


\title{
High expression of the circadian gene $m$ Per2 diminishes the radiosensitivity of NIH 3T3 cells
}

\author{
L. Chang, Y.Y. Liu, B. Zhu, Y. Li, H. Hua, Y.H. Wang, \\ J. Zhang, Z. Jiang and Z.R. Wang \\ Health Ministry Key Laboratory of Chronobiology, \\ West China Medical Center, Sichuan University, Chengdu, China
}

\begin{abstract}
Period2 is a core circadian gene, which not only maintains the circadian rhythm of cells but also regulates some organic functions. We investigated the effects of $m P e r i o d 2(m P e r 2)$ expression on radiosensitivity in normal mouse cells exposed to ${ }^{60} \mathrm{Co}-$ $\mathrm{Y}$-rays. NIH 3T3 cells were treated with 12-O-tetradecanoylphorbol-13-acetate (TPA) to induce endogenous $m P e r 2$ expression or transfected with pcDNA3.1(+)-mPer2 and irradiated with ${ }^{60} \mathrm{Co}-\mathrm{y}$-rays, and then analyzed by several methods such as flow cytometry, colony formation assay, RT-PCR, and immunohistochemistry. Flow cytometry and colony formation assay revealed that irradiated NIH 3T3 cells expressing high levels of mPer2 showed a lower death rate (TPA: $24 \mathrm{~h} 4.3 \%$ vs $12 \mathrm{~h} 6.8 \%$ and control 9.4\%; transfection: pcDNA3.1-mPer2 3.7\% vs pcDNA3.1 11.3\% and control 8.2\%), more proliferation and clonogenic survival (TPA: $121.7 \pm 6.51$ vs $66.0 \pm 3.51$ and $67.7 \pm 7.37$; transfection: $121.7 \pm 6.50$ vs $65.3 \pm 3.51$ and $69.0 \pm 4.58$ ) both when treated with TPA and transfected with mPer2. RT-PCR analysis showed an increased expression of bax, bcl-2, p53, c$m y c, m r e 11$, and $n b s 1$, and an increased proportionality of $b c /-2 / b a x$ in the irradiated cells at peak $m P e r 2$ expression compared with cells at trough mPer2 expression and control cells. However, no significant difference in rad50 expression was observed among the three groups of cells. Immunohistochemistry also showed increased protein levels of P53, BAX and proliferating cell nuclear antigen in irradiated cells with peak $m P e r 2$ levels. Thus, high expression of the circadian gene mPer2 may reduce the radiosensitivity of $\mathrm{NIH} 3 \mathrm{~T} 3$ cells. For this effect, $m P e r 2$ may directly or indirectly regulate the expressions of cell proliferation- and apoptosis-related genes and DNA repair-related genes.
\end{abstract}

Key words: Circadian; mPer2; Radiation; Cell death; Proliferation; DNA repair

\section{Introduction}

Radiobiology studies have shown that ionizing radiation is a DNA-damaging agent inducing cell death, gene mutations and chromosome aberrations even at low doses (1). Most of these reactions are induced by hydroxyl radicals (indirect effects) and by one-electron oxidation (direct effects) resulting from exposure to ionizing radiation (2). Cell DNA molecules are important targets of radiation injury $(3,4)$. Activation of the damage checkpoint occurs in response to many types of genomic lesions, including double-strand DNA breaks, single-strand DNA breaks and chemical modification of DNA by UV and $y$ irradiation $(5,6)$. The cell cycle potentially stalls at several phases to provide ample time for the cell to repair DNA lesions before the S-phase (G1 arrest) and/or mitosis (G2 arrest). When cells recognize
DNA injuries, especially double-strand DNA breaks, they activate the DNA damage checkpoint and repair the damage. When cells fail to fully repair the disordered DNA they activate the apoptotic cell death pathways. This fine tuning of the balance between DNA repair and apoptosis may be mediated by the DNA-binding properties of the related proteins and by their transactivation of gene transcription such as the P53 protein (7-9).

Circadian genes constitute the physiological basis of circadian clocks, which control the daily oscillations of biochemical, physiological, and behavioral processes of most organisms and enable these processes to occur at appropriate times of day (10-12). Recently, it has been shown that circadian genes not only play an important

Correspondence: Z.R. Wang, Health Ministry Key Laboratory of Chronobiology, West China Medical Center, Sichuan University, No.17 Section 3 South Renming Road, Chengdu, 610041, China. Fax: +86-028-8550-3204. E-mail: wangzhengrong@126.com

Research supported by the China National Natural Science Foundation (Nos. 30871357 and 30700393) and by CMB (No. 88-486). Received January 23, 2009. Accepted July 22, 2009. Available online September 4, 2009. 
role in controlling circadian rhythms, but also participate in other physiological and pathological activities, such as drug dependence, tumor development and radiation response (13-15).

The Period2 (Per2) gene, an indispensable component of the circadian clock, not only modulates circadian oscillations, but also regulates other organic functions. mPeriod2 (mPer2) gene-deficient mice are cancer prone. After $\mathrm{Y}$ radiation, these mice showed a marked increase in tumor development and reduced thymocyte apoptosis. Temporal expression of the genes involved in cell cycle regulation and tumor suppression, such as Cyclin D1, Cyclin A, Mdm2 , and Gadd45, were reportedly altered in $m P e r 2$ mutant mice (16). The mPer2 gene may play an important role in tumor suppression by regulating DNA damage-response pathways.

It previous studies from our laboratory, Zhang et al. (17) observed that high expression of the circadian gene $m P e r 2$ might reduce the radiosensitivity of irradiated mouse tumor cells such as LLC and EMT6 cells. In the present study, we focus on the effects of the circadian gene mPer2 on irradiated normal cells such as NIH 3T3 cells. We treated NIH 3T3 cells with 12-O-tetradecanoylphorbol-13-acetate (TPA) to induce endogenous circadian mPer2 expression, or established mPer2-overexpressing cells by transfecting pcDNA3.1(+)-mPer2 into NIH 3T3 cells. We then exposed the cells to ${ }^{60} \mathrm{Co}-\mathrm{y}$-rays, assessed the effects of mPer2 on cell death, proliferation and clonogenic survival after radiation, and explored the possible mechanism involved. We trust that the present study will offer a new theory for radiochronotherapy and provide a new way to protect normal cells against radiation injury.

\section{Material and Methods}

\section{Cell culture}

NIH 3T3 cells were maintained in Dulbecco's modified Eagle's medium (DMEM; Hyclone, USA) supplemented with antibiotics (BioWhitaker, USA) and 10\% fetal calf serum (Hyclone) in a humidified atmosphere of $95 \%$ air and $5 \%$ $\mathrm{CO}_{2}$ at $37^{\circ} \mathrm{C}$.

\section{Induction of rhythm by TPA treatment}

TPA, the classic protein kinase $\mathrm{C}$ activator, was purchased from Promega (USA). We treated NIH 3T3 cells with $100 \mathrm{nM}$ TPA to induce endogenous circadian $m P e r 2$ expression. At time zero, TPA was added to the medium, which was replaced with serum-free DMEM after $2 \mathrm{~h}$. TPA treatment without serum can trigger the induction of the circadian oscillation of expression of some genes, including $m$ Per2, with an approximate period of $24 \mathrm{~h}$ in NIH 3T3 cells. And the trough level of mPer 2 mRNA occurs $12 \mathrm{~h}$ after TPA treatment and peaks $24 \mathrm{~h}$ after TPA treatment (18). Cells were then divided into three radiotreatment groups: a) control group (cells irradiated without TPA treatment), b) TPA 12-h group (cells irradiated at $12 \mathrm{~h}$ after TPA treatment with trough mPer2 level), and c) TPA24-h group (cells irradiated at $24 \mathrm{~h}$ after TPA treatment with peak mPer2 level).

\section{Cell transfection}

The eukaryotic expression vector pcDNA3.1(+)mPer2 containing a cDNA copy of mPer2 (GenBank No. NM_011066) was used in this study. The mPer2 gene was confirmed as being in frame with no mutations by DNA sequencing. The cells were transfected with the indicated plasmids using the lipofectamine 2000 transfection reagent (Invitrogen, USA). Cell lysates were prepared $48 \mathrm{~h}$ later for the examination of protein expression and radiotreatment. The cells were divided into three groups: a) control group (cells without transfection), b) pcDNA3.1 group (cells transfected with the empty vector pcDNA3.1), and c) pcDNA3.1mPer2 group (cells transfected with pcDNA3.1-mPer2).

\section{Antibodies}

Mouse antibodies against mPER2, P53, BAX and proliferating cell nuclear antigen (PCNA) were purchased from Sigma (USA). Rabbit anti-goat IgG and horseradish peroxidase-conjugated secondary antibodies were purchased from Santa Cruz Biotechnology (USA).

\section{Western blot analysis}

At $48 \mathrm{~h}$ after transfection, cells were lysed with cold RIPA lysis buffer (Sigma-Aldrich, USA) containing protease inhibitors, and proteins were collected by centrifugation. Protein concentrations were determined by the bicinchoninic acid assay (Pierce, USA) and transferred electro-phoretically to a polyvinylidene difluoride membrane (Pierce). Detection was carried out using an enhanced chemiluminescence reagent (Pierce).

\section{Radiotreatment}

All cell groups were irradiated with $y$-rays, with a total absorbed dose of $4 \mathrm{~Gy}$, using a ${ }^{60} \mathrm{Co}$ teletherapy machine (Phoenix, Japan). Cells were exposed at a dose rate of $115.38 \mathrm{cGy} / \mathrm{min}$ in an exposure field of $25 \times 25 \mathrm{~cm}$, with spacing of $80 \mathrm{~cm}$. Cells were then processed for flow cytometry and colony formation assay to test cell death, proliferation and clonogenic survival.

\section{Flow cytometry}

To determine the expression of mPER2 protein, the cells were harvested at $48 \mathrm{~h}$ after transfection, fixed in $70 \%$ ethanol for $30 \mathrm{~min}$ at $4^{\circ} \mathrm{C}$, and incubated with $0.1 \%$ saponin for $20 \mathrm{~min}$. They were then incubated with primary antibodies (Sigma) at 1:200 dilution for $30 \mathrm{~min}$ and with fluoresceinisothiocyanate-conjugated secondary antibodies (Chemicon, USA) at 1:150 dilution for $30 \mathrm{~min}$, and analyzed by flow cytometry (Beckman Coulter Elite ESP, USA).

Six hours after radiotreatment, all cells were digested with trypsin, harvested, washed with phosphate-buffered 
saline (PBS), fixed in $70 \%$ ethanol for $30 \mathrm{~min}$ at $4{ }^{\circ} \mathrm{C}$, treated with $50 \mu \mathrm{g} / \mathrm{mL}$ RNase A (Sigma), stained with $50 \mu \mathrm{g} / \mathrm{mL}$ propidium iodide for $20 \mathrm{~min}$ at $4^{\circ} \mathrm{C}$ without light, and analyzed by flow cytometry using an instrument equipped with a 488-nm argon laser for the determination of DNA synthesis and cell cycle status. Data were collected in linear mode and analyzed with the Multicycle Software (Beckman Coulter, USA). Apoptotic cells with degraded DNA appear as cells with hypodiploid DNA content and are represented in socalled "sub-G1" peaks on DNA histograms. Four distinct phases were recognized by flow cytometry in a proliferating cell population, including the G0/G1, S- (DNA synthesis phase), G2 and M-phases (mitosis).

\section{Colony formation assay}

Six hours after radiotreatment, all cells were digested with trypsin, statically cultured in DMEM in the cell culture incubator (SANYO, Japan) for 14 days, washed with PBS, fixed with methanol, stained for $15 \mathrm{~min}$ and washed again. The preparation was then photographed with the Omegapic formatter and analyzed.

\section{RT-PCR}

Total RNA of cells treated by TPA was isolated with Trisol reagent (Invitrogen) 30 min after irradiation. RT-PCR for mouse bax, bcl-2, p53, c-myc, rad50, mre11, nbs1, and GAPDH mRNA was carried out. The primer sequences were 5'-GATGCGTCCACCAAGAA-3' and 5'-AGTAGAAG AGGGCAACCAC-3' for bax, 5'-CCCAAGGGAAGACG ATG-3' and 5'-GAGCGGGTAGGGAAAGA-3' for $b c /-2$, 5'-CCCAAGGGAAGACGATG-3' and 5'-GAGCGGGTAG GGAAAGA-3' for c-myc, 5'-GCAACGAGCCCTCAACA-3' and 5'-GGACCCACGGATGAACCT-3' for p53, 5'-TTTGG CGGAGTACCTATC-3' and 5'-CACCACTCGGTAGTTGT AAT-3' for rad50, 5'-GGCGAAGCAGTTCAAGAG-3' and 5'-GGCTGTTGTCGGGTAGAT-3' for mre 11, 5'-GGAAGCC GACACCTCATC-3' and 5'-CACAATCATTTACGCACAG-3' for $n b s 1$, and 5'-TCACTGCCACCCAGAAGA-3' and 5'-AAGTCGCAGGAGACAACC-3' for GAPDH. RT-PCR products were detected by $1 \%$ agarose electrophoresis and analyzed according to the integral optical density method with a Gel-Pro analyzer. Lane-to-lane variation in the amount of loaded mRNA was controlled internally by normalizing the level of each gene to that of GAPDH.

\section{Immunohistochemistry}

Six hours after irradiation, the cells treated with TPA were used for immunohistochemistry. The test was carried out using the avidin-biotin complex method. The numbers of P53-, BAX- and PCNA-positive and -negative NIH 3T3 cells were determined in four random fields at $100 \mathrm{X}$ and 400X magnification, and the percentages of positive cells were calculated.

\section{Statistical analysis}

Data are reported as means \pm SD. One-way ANOVA was used to compare difference among groups and $P$ values of less than 0.05 were considered to be statistically significant.

\section{Results}

The effects of radiation on NIH 3T3 cells treated with TPA

Parental NIH 3T3 cells normally produce very low and barely detectable levels of mPer2. In the present study, NIH 3T3 cells, TPA treatment was used to induce endogenous mPer2 expression and to trigger the induction of the circadian oscillation of expression of various clock and clock-related genes, including $m P e r 2$. The expression level of $m$ Per2 mRNA oscillated within an approximate period of $24 \mathrm{~h}$, with the trough occurring after $12 \mathrm{~h}$ and the peak after $24 \mathrm{~h}$ of TPA treatment.

The death and proliferation of cells irradiated after TPA treatment were determined by flow cytometry. The cells irradiated at peak $m$ Per2 expression had much lower apoptotic peaks than cells irradiated at trough $m P e r 2$ expression and than control cells irradiated without TPA treatment $(\mathrm{P}<$ 0.01; Figure 1).
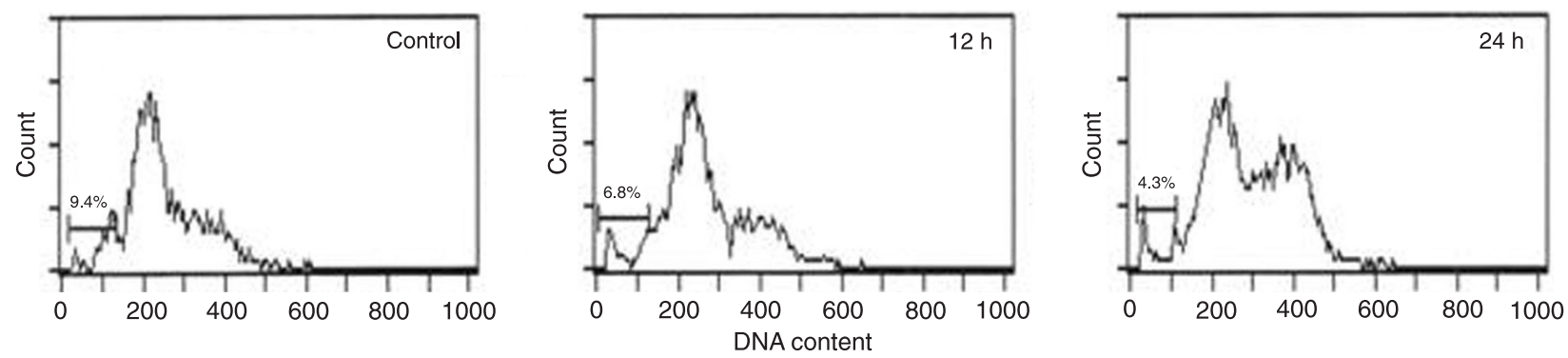

Figure 1. Apoptosis in NIH 3T3 cells irradiated after 12-O-tetradecanoylphorbol-13-acetate (TPA) treatment. Control: cells irradiated without TPA treatment; $12 \mathrm{~h}$ : cells irradiated $12 \mathrm{~h}$ after TPA treatment; $24 \mathrm{~h}$ : cells irradiated $24 \mathrm{~h}$ after TPA treatment. The percentage of sub-G1 cells undergoing apoptosis is indicated by the bar. The cells of the TPA 24-h group had a significantly lower apoptotic peak than the cells of the TPA 12-h group and of control cells ( $P<0.01$, one-way ANOVA). 
The G0/G1, S-, G2, and M-phases of the cell cycle were also analyzed by flow cytometry, with the following cell percentages being observed in each phase: control group, G0/G1 (59.3 $\pm 1.07 \%)$, S (29.2 $\pm 0.30 \%)$, and G2/M (11.7 $\pm 0.21 \%$ ); TPA 12-h group, G0/G1 (58.8 \pm $0.91 \%), S(32.2 \pm 0.66 \%)$ and G2/M (9.4 $\pm 0.47 \%)$; TPA 24-h group, G0/G1 (53.2 $\pm 0.73 \%)$, S (42.7 $\pm 0.43 \%)$, and $\mathrm{G} 2 / \mathrm{M}(4.1 \pm 0.34 \%)$. The $\mathrm{S}$-phase fraction was greater in TPA 24-h group cells than in TPA 12-h group cells and control cells ( $P<0.01$; Figure 2$)$. Clonogenic survival was determined by the colony formation assay. The colony-forming efficiency was significantly higher in TPA 24-h group cells than in TPA 12-h group cells and control cells $(P<0.01$; Figure 3 ). Irradiated $\mathrm{NIH}$
3T3 cells expressing high levels of $m$ Per2 showed less cell death and more cell proliferation and clonogenic survival. Three independent experiments demonstrated similar results.

\section{Role of radiation in NIH 3T3 cells with up-regulated mPer2}

We established mPer2-overexpressing cells by transfecting pcDNA3.1(+)-mPer2 into NIH 3T3 cells. Successful transfection of the mPer2 gene using the lipofectamine 2000 reagent was confirmed by Western blotting (Figure 4A). It was also evident by flow cytometry that the fluorescence intensity of mPER2 protein expression in pcDNA3.1(+)-mPer2-transfected cells was
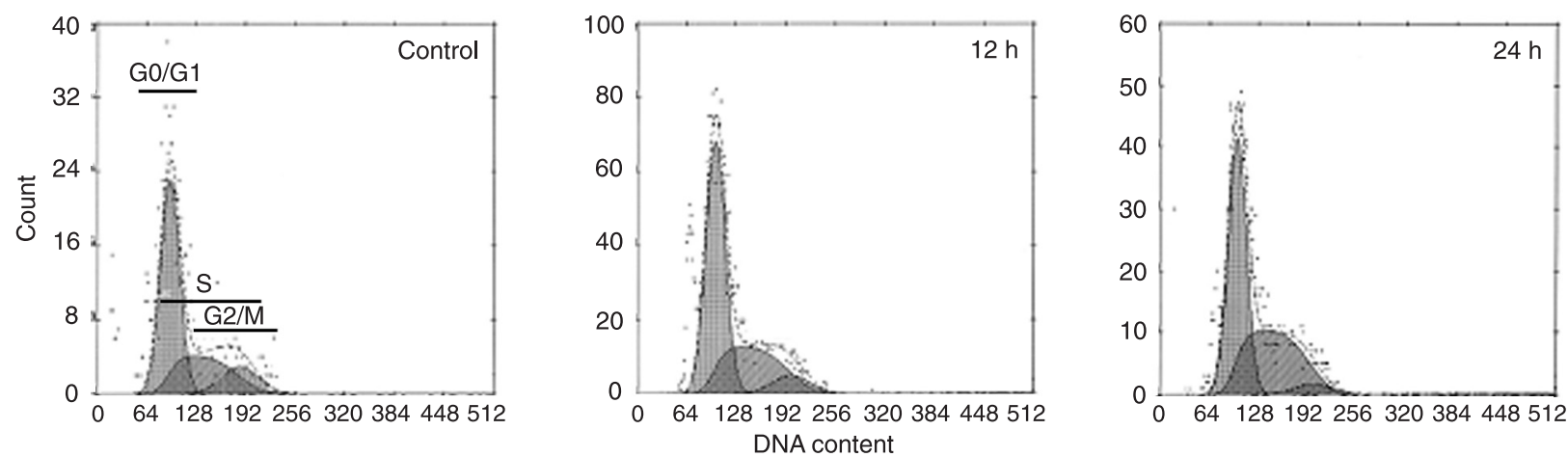

Figure 2. The cell cycle in NIH 3T3 cells irradiated after 12-O-tetradecanoylphorbol-13-acetate (TPA) treatment. Control: cells irradiated without TPA treatment; $12 \mathrm{~h}$ : cells irradiated $12 \mathrm{~h}$ after TPA treatment; $24 \mathrm{~h}$ : cells irradiated $24 \mathrm{~h}$ after TPA treatment. G0/G1, S and G2/M phases are indicated. The S-phase fraction of the TPA 24-h group was significantly higher than that of the TPA 12-h group and of control cells $(P<0.01$, one-way ANOVA).

Figure 3. Colony formation of 12-O-tetradecanoylphorbol-13acetate (TPA)-treated NIH 3 T3 cells after irradiation. $A$, Control: cells irradiated without TPA treatment; $12 \mathrm{~h}$ : cells irradiated $12 \mathrm{~h}$ after TPA treatment; $24 \mathrm{~h}$ : cells irradiated $24 \mathrm{~h}$ after TPA treatment. $B$, High expression of mPER2 dramatically increased the clonogenic survival of irradiated NIH 3T3 cells. The colony-forming efficiency of TPA 24-h group cells was significantly higher than that of TPA 12-h group cells and of control cells $(P<0.01$, one-way ANOVA).

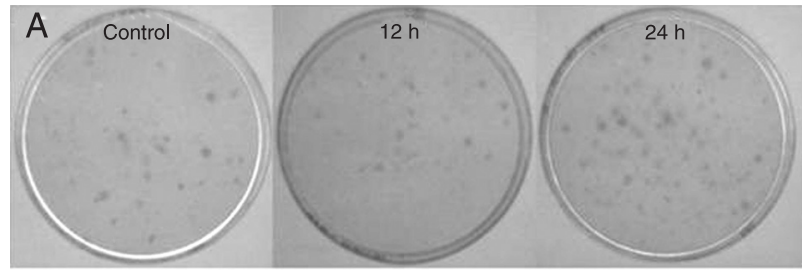

B

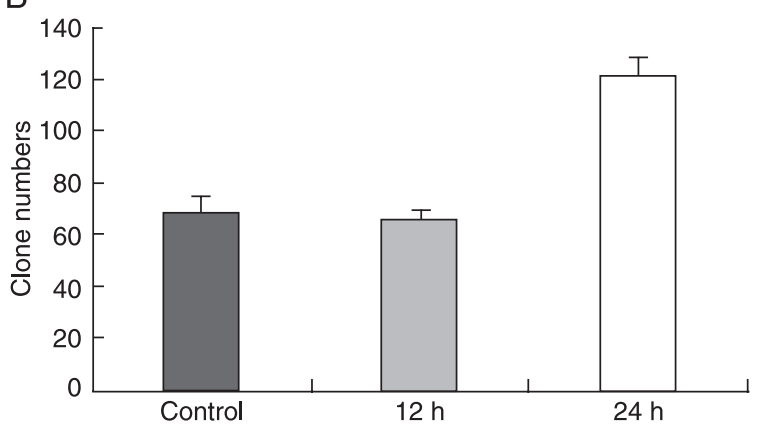


higher than in pcDNA3.1-transfected cells and in control cells without transfection (Figure 4B).

The effect of mPer2 on the death, proliferation and clonogenic survival of cells irradiated after transfection was determined by flow cytometry and the colony formation assay. The results revealed that the apoptotic peak was lower in $m$ Per2 overexpressing cells than in empty vector cells and control cells ( $P<0.01$; Figure 5$)$. Analysis of cell cycle distribution showed: control group, G0/G1 (46.3 $\pm 1.21 \%)$, S $(40.9 \pm 1.67 \%)$, and G2/M (12.8 $\pm 0.78 \%)$; pcDNA3.1 group, G0/G1 (55.6 $\pm 0.84 \%), \mathrm{S}(39.0 \pm 0.56 \%)$, and G2/M (5.4 \pm 0.41\%); pcDNA3.1-mPer2 group, G0/G1 (45.9 \pm $1.04 \%), S(46.5 \pm 0.72 \%)$, and G2/M (7.6 $\pm 0.54 \%)$. The S-phase fraction of $m$ Per2 overexpressing cells was higher than that of empty vector cells and control cells $(P<0.01$; Figure 6). mPER2 dramatically increased the clonogenic survival of irradiated NIH 3T3 cells. The colony-forming efficiency of $m P e r 2$ overexpressing cells was also higher than that of other cells $(P<0.01$; Figure 7$)$. Three independent

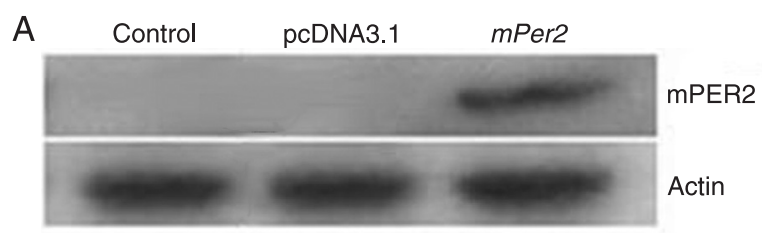

B

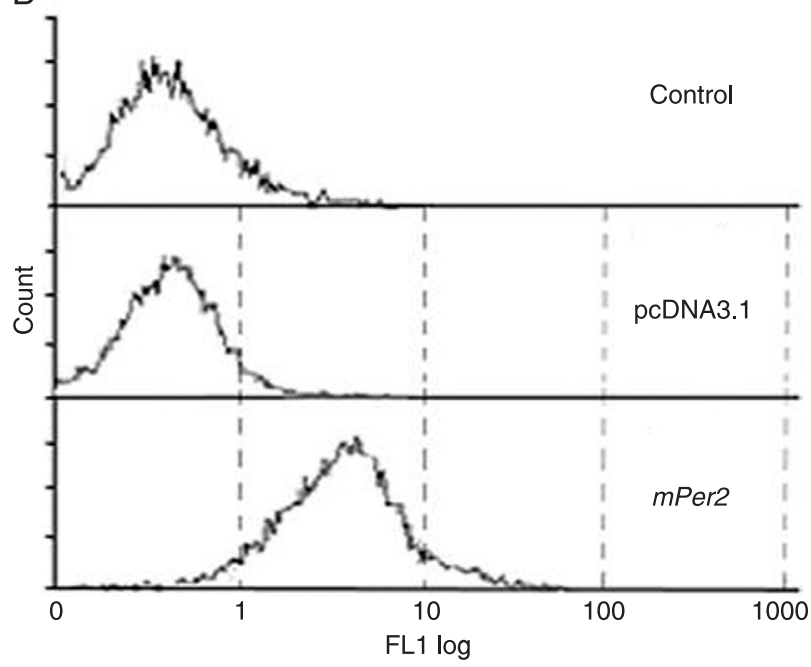

Figure 4. Detection of mPER2 expression by Western blot analysis and flow cytometry. Control: cells without transfection; pcDNA3.1: cells transfected with pcDNA3.1; mPer2: cells transfected with pcDNA3.1-mPer2. A, Western blot analysis of mPER2 expression. The mPER2 expression of $m P e r 2$ group cells was significantly higher than that of pcDNA3.1 group cells and of control cells. $B$, Flow cytometric analysis of mPER2 expression. The fluorescence intensity of mPER2 expression in mPer2 group cells was also significantly higher than that of pcDNA3.1 group cells and of control cells $(P<0.01$, one-way ANOVA). experiments demonstrated that irradiated NIH 3 T3 cells overexpressing mPer2 also showed less cell death and more cell proliferation and clonogenic survival.

\section{mPer2 up-regulated the expressions of cell proliferation- and apoptosis-related genes and DNA repair-related genes and proteins}

We chose RT-PCR analysis and immunohistochemistry to study the mechanism of the effects of mPer2 gene expression on the response of NIH 3 T3 cells to radiation. RT-PCR analysis showed that the mRNA levels of bax, $b c l-2, p 53, c-m y c$, mre11, and nbs1 were up-regulated in the irradiated cells at peak mPer2 expression compared with cells at trough mPer 2 expression and control cells, with the expression of $c-m y c$ and $b c l-2$ being particularly up-regulated. However, there was no significant difference in rad50 level among the three cell groups (Figure 8). Immunohistochemistry also showed increased protein levels of P53, BAX and PCNA in irradiated NIH 3T3 cells with high mPER2 expression (Figure 9). Percentages of positive cells were: P53, control (34.1 $\pm 2.7 \%), 12 \mathrm{~h}(40.2 \pm 3.9 \%)$, and $24 \mathrm{~h}(61.3 \pm 3.6 \%)$; BAX, control $(20.7 \pm 4.1 \%), 12 \mathrm{~h}(22.8$ $\pm 4.3 \%)$, and $24 \mathrm{~h}(37.1 \pm 6.4 \%) ;$ PCNA, control $(29.4 \pm$ $3.2 \%), 12$ h $(33.7 \pm 5.6 \%)$, and 24 h $(87.3 \pm 4.7 \%)$. Three independent experiments demonstrated similar results.

\section{Discussion}

Circadian clocks are molecular time-keeping mechanisms that reside in a diverse range of cell types in a variety of organisms. The primary role of these cell-autonomous clocks is to maintain their own approximately 24-h molecular rhythms and to drive the rhythmic expression of genes involved in physiology, metabolism and behavior $(19,20)$. The circadian clock is composed of multiple, single-cell circadian oscillators, which, when synchronized, generate coordinated circadian outputs that regulate overt rhythms. Eight clock genes involved in the interacting transcriptional-/ translational-feedback loops that compose the molecular clockwork have been cloned (21).

Parental NIH 3T3 fibroblasts normally produce very low and barely detectable levels of circadian genes. TPA treatment is as effective as serum shock in triggering the induction of circadian gene expression in cultured cells, including $m P e r 2$. As a core circadian gene, $m$ Per2 not only maintains the circadian rhythm of cells, but also sustains the normal cell cycle. After TPA treatment, parental NIH 3T3 cells present circadian oscillation of $m P e r 2$ expression within an approximate period of $24 \mathrm{~h}$, with the trough at zeitgeber time 12 (ZT12)and the peak at ZT24 $(18,22,23)$.

In the present study, we treated NIH 3 T3 cells with TPA to induce endogenous $m P e r 2$ expression, and cells were irradiated at ZT12 with trough $m P e r 2$ level and at ZT24 with peak $m P e r 2$ level. The death, proliferation and clonogenic survival of irradiated cells were determined by flow cytom- 

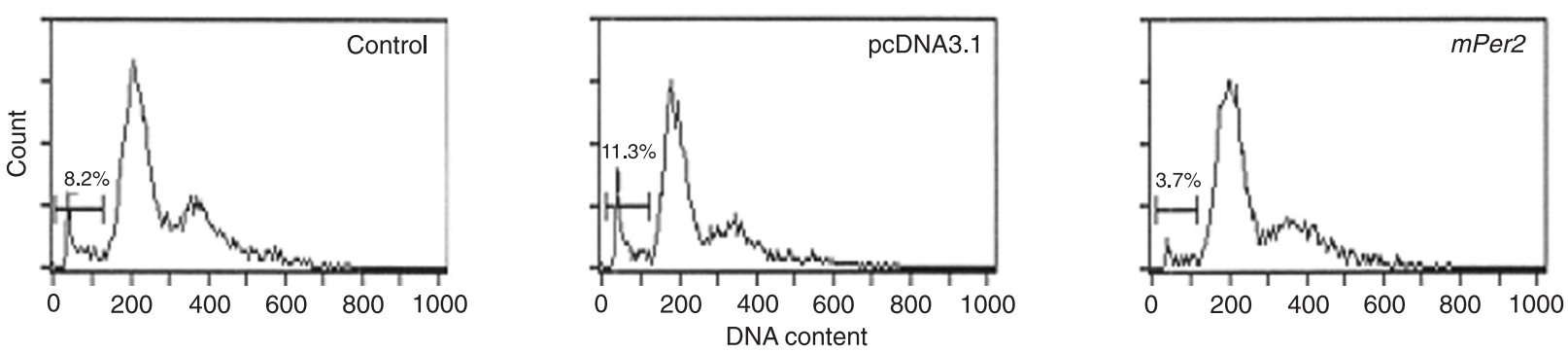

Figure 5. Apoptosis in NIH 3T3 cells irradiated after transfection. Control: cells without transfection; pcDNA3.1: cells transfected with pcDNA3.1; mPer2: cells transfected with pcDNA3.1-mPer2. The percentage of sub-G1 cells undergoing apoptosis is indicated by the bar. The apoptotic peak of $m P e r 2$ overexpressing cells was lower than that of empty vector cells and of control cells $(P<0.01$, one-way ANOVA).
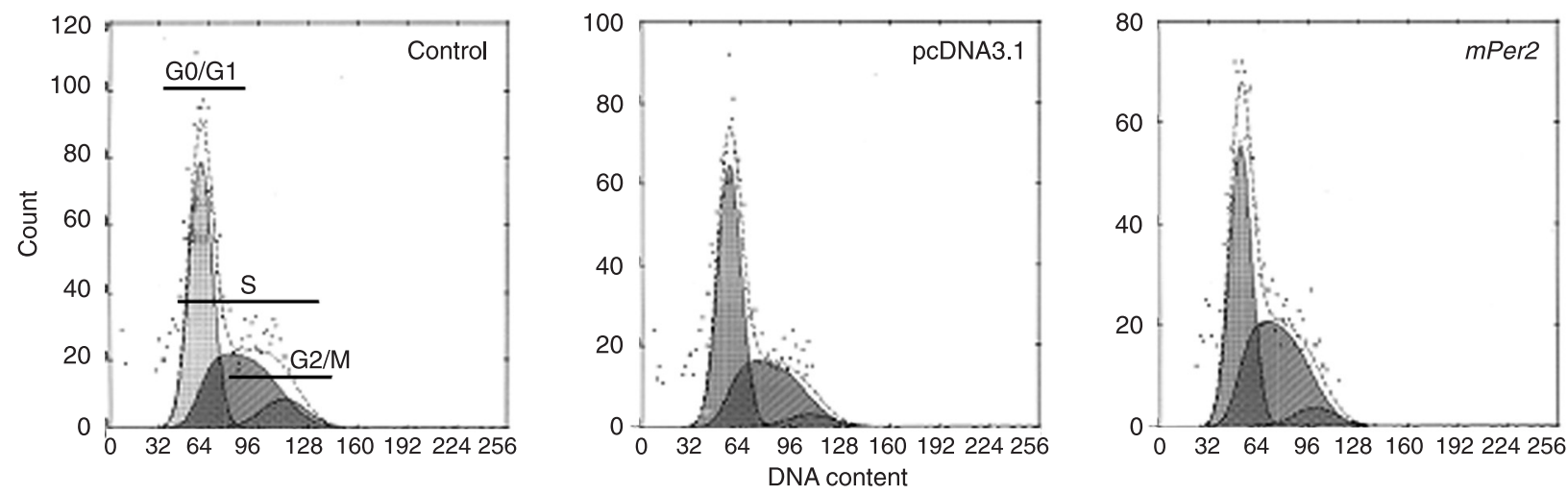

Figure 6. The cell cycle in NIH 3T3 cells irradiated after transfection. Control: cells without transfection; pcDNA3.1: cells transfected with pcDNA3.1; mPer2: cells transfected with pcDNA3.1-mPer2. G0/G1, S and G2/M phases are indicated. The S-phase fraction of $m$ Per2 overexpressing cells was higher than that of empty vector cells and of control cells $(P<0.01$, one-way ANOVA).

Figure 7. Colony formation of transfected NIH 3 T3 cells after irradiation. $A$, Control: cells without transfection; pcDNA3.1: cells transfected with pcDNA3.1; mPer2: cells transfected with pcDNA3.1-mPer2. B, mPER2 dramatically increased the clonogenic survival of irradiated NIH 3 T3 cells. The colony-forming efficiency of $m P e r 2$ overexpressing cells was significantly higher than that of empty vector cells and of control cells $(P<0.01$, one-way ANOVA).
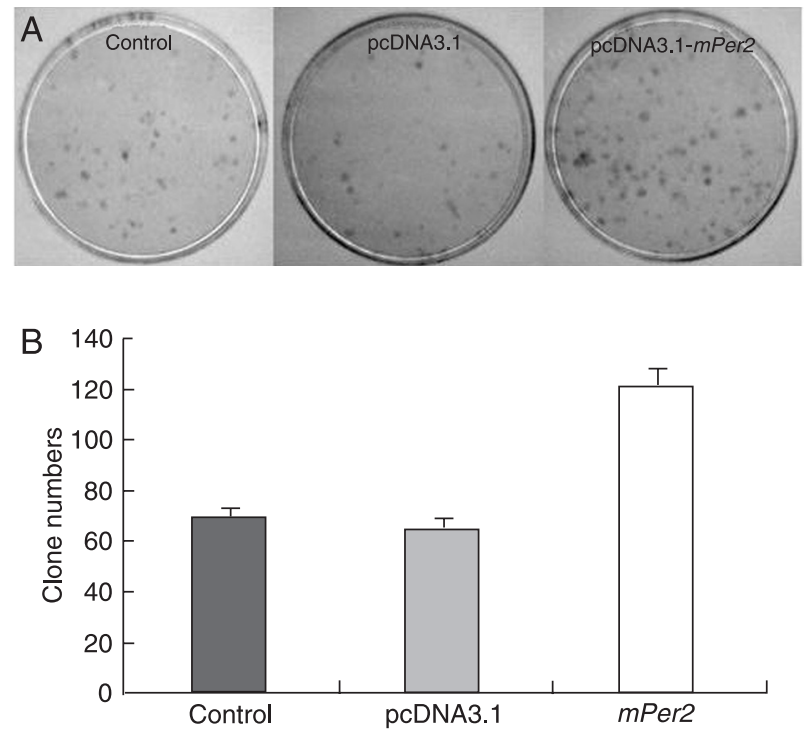

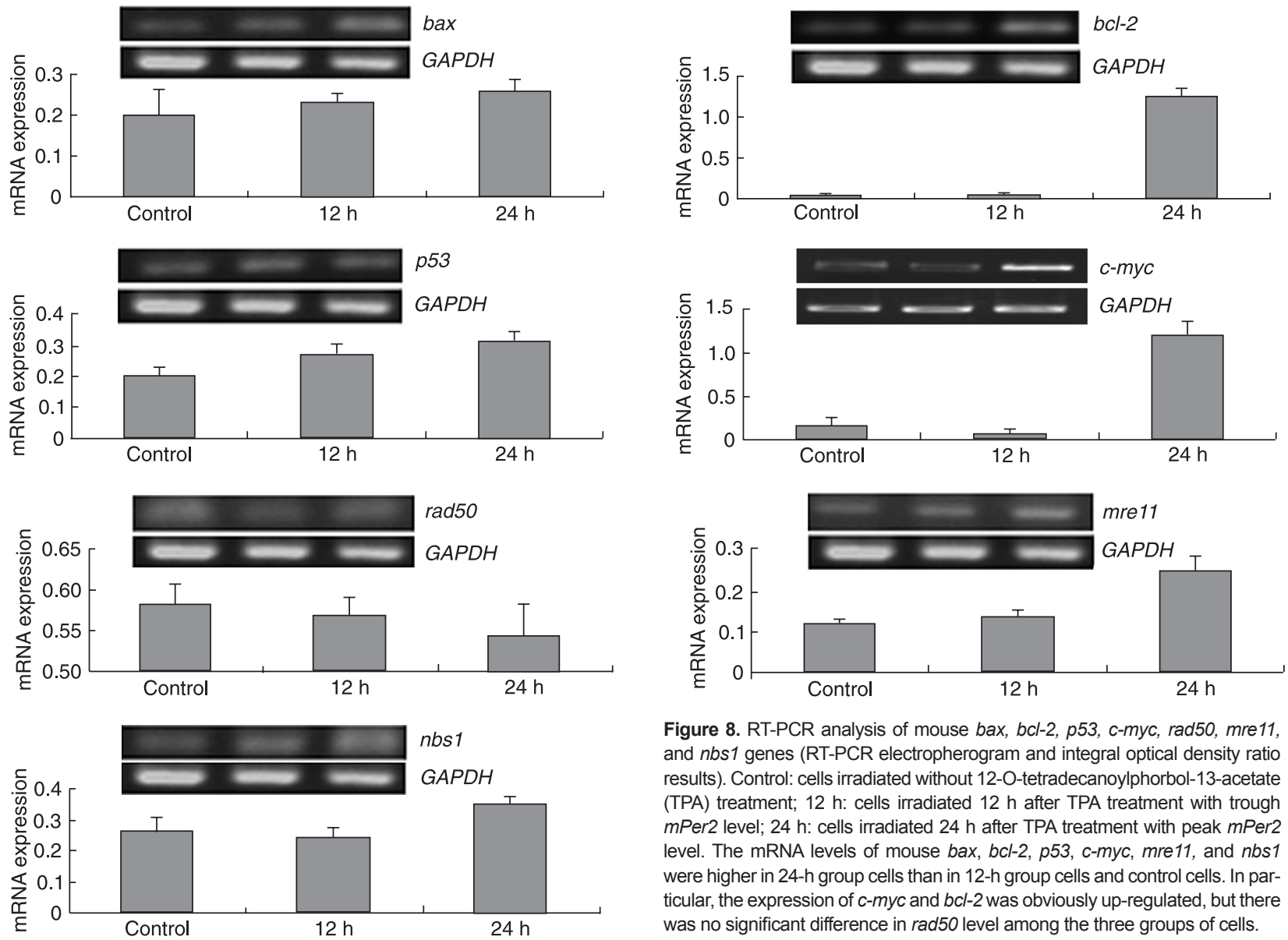

Figure 8. RT-PCR analysis of mouse bax, bcl-2, p53, c-myc, rad50, mre11, and $n b s 1$ genes (RT-PCR electropherogram and integral optical density ratio results). Control: cells irradiated without 12-O-tetradecanoylphorbol-13-acetate (TPA) treatment; $12 \mathrm{~h}$ : cells irradiated $12 \mathrm{~h}$ after TPA treatment with trough $m$ Per2 level; $24 \mathrm{~h}$ : cells irradiated $24 \mathrm{~h}$ after TPA treatment with peak $m P e r 2$ level. The mRNA levels of mouse bax, bcl-2, p53, c-myc, mre11, and nbs1 were higher in 24-h group cells than in 12-h group cells and control cells. In particular, the expression of $c-m y c$ and $b c l-2$ was obviously up-regulated, but there was no significant difference in rad50 level among the three groups of cells.

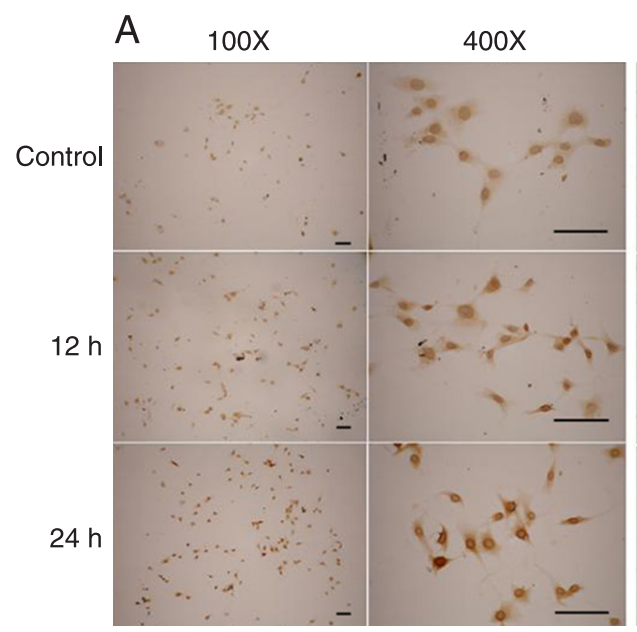

B $100 x$

$400 X$

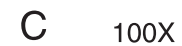

$400 X$
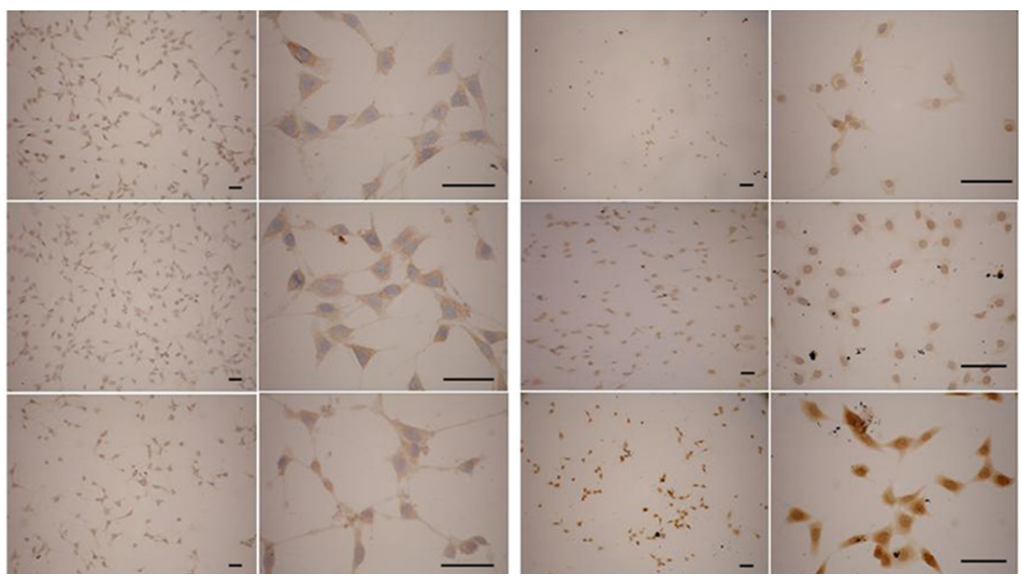

Figure 9. Expression of $\mathrm{P} 53(\mathrm{~A}), \mathrm{BAX}(\mathrm{B})$ and proliferating cell number antigen (PCNA; $)$ ) proteins determined by immunocytochemistry (100X, 400X magnification). Control: cells irradiated without 12-O-tetradecanoylphorbol-13-acetate (TPA) treatment; $12 \mathrm{~h}$ : cells irradiated $12 \mathrm{~h}$ after TPA treatment; $24 \mathrm{~h}$ : cells irradiated $24 \mathrm{~h}$ after TPA treatment. P53, BAX and PCNA levels were up-regulated in cells irradiated with peak $m P e r 2$ level (24-h group) compared to cells irradiated with trough $m$ Per2 level (12-h group) and control cells. Magnification bar $=200 \mu \mathrm{m}$. 
etry and colony formation assay. The results of the present study showed that high expression of $m P e r 2$ might reduce cell death and enhance cell proliferation of irradiated NIH 3T3 cells. However, after TPA treatment, parental NIH 3T3 cells presented circadian expression not only of $m P e r 2$, but also of other clock and clock-related genes such as mPer1 and $D B P(18)$. Therefore, the radiobiological effects may result from $m P e r 2$ or other clock and clock-related genes. In order to assess the effects of mPer2 expression on the irradiated cells, we transfected pcDNA3.1(+)-mPer2 into $\mathrm{NIH} 3 \mathrm{~T} 3$ cells. The results showed that overexpression of mPER2 in NIH 3T3 cells resulted in reduced cell death and enhanced cell proliferation after radiation, suggesting that overexpressed $m P e r 2$ may diminish the radiosensitivity of NIH 3 T3 cells.

Fu et al. (16) reported that the loss of mPer2 function resulted in increased tumor development and deficiencies in response to DNA damage in mice, suggesting that the $m$ Per2 gene functions in tumor suppression by regulating DNA damage-responsive pathways. Compared with wildtype mice, mPer2 mutant mice showed a neoplastic growth phenotype and an increased sensitivity to $y$ radiation in thymocytes. Zhang et al. (17) also found that high expression of the circadian gene $m P e r 2$ might diminish radiosensitivity of irradiated mouse tumor cells. LLC and EMT6 cells with high $m P e r 2$ expression exposed to ${ }^{60} \mathrm{Co}-\mathrm{Y}$-rays presented reduced DNA damage, increased survival and clone-forming rate, which suggested that $m P e r 2$ might protect cells against the radiation injury of $\mathrm{y}$-rays and increase the survival rate of tumor cells. The present study demonstrated that high expression of mPer2 could diminish the radiosensitivity of NIH 3T3 cells, with reduced cell death, enhanced cell proliferation and increased clonogenic survival. Moreover, tumor cells and NIH 3T3 cells were irradiated at different times after TPA induction, i.e., at $12 \mathrm{~h}$ (trough time of $m P e r 2$ expression) and at $24 \mathrm{~h}$ (peak time) after induction, whereas LLC cells were irradiated at $18 \mathrm{~h}$ (trough time) and $30 \mathrm{~h}$ (peak time) after induction. The present research combined with the previous research of Fu et al. (16) and Zhang et al. (17) would contribute to protecting normal cells against radiation injury in cancer radiotherapy. We propose that radiotherapy could be used against cancer at a certain time of Per2 expression for the best ratio of tumor suppression and normal tissue protection, which can kill tumor cells and protect normal cells maximally. However, our hypothesis needs further study.

Without radiation, overexpression of $\mathrm{mPER} 2$ results in reduced proliferation and rapid apoptosis of tumor cells, but not of NIH 3T3 cells, suggesting that mPer2 may play an important role in tumor suppression by inducing apoptotic cell death (24). However, irradiated NIH 3T3 cells with mPER2 overexpression showed reduced cell death, suggesting that $m$ Per2 may affect the radiation-induced cell death by other ways.

In response to ionizing radiation, cells immediately activate a series of biochemical pathways that promote cell survival while maintaining genetic integrity. The main cellular defense system against ionizing radiation exposure is composed of two distinct types of biochemical pathways, i.e., the DNA damage cell cycle checkpoint pathways and the DNA repair pathways (25). Arrest of replicative DNA synthesis after DNA damage is thought to occur to provide ample time for the cell to repair DNA lesions before the Sphase (G1 arrest) and/or mitosis (G2 arrest).

The RAD50-MRE11-NBS1 (MRN) complex plays an important role in the repair of DNA damage caused by radiation. The complex practically participates in all repair mechanisms, especially homologous recombination and non-homologous end-joining, which are the most important mechanisms in the repair of DNA double-strand breaks (26). In the present study, the levels of rad50 expression of the various cell groups were not different, in agreement with reports showing that rad50 expression is persistent and stable (27). As the core part of the MRN complex, MRE11 has not only nuclease activity, but also a connective effect on RAD50 and NBS1, and its expression changes rapidly after radiation (28). NBS1 is also an important part of the MRN complex (29). The irradiated NIH 3T3 cells expressing high levels of $m P e r 2$ showed an increased expression of mre $1 /$ and $n b s 1$, which suggested that $m P e r 2$ could enhance the function of DNA damage repair MRN complex.

$c-M y c$, a proto-oncogene, plays an important role in both cell proliferation and apoptosis $(30,31)$. Circadian regulators may target genes that are controlled by c-myc. c-Myc itself is also controlled by the circadian clock, and the level of c-myc mRNA oscillated in 24-h light/dark cycles in wild-type mouse livers, peaking at ZT14 (16). Gamma radiation may increase the expression of $c-m y c$, whose overexpression could drive cells to progress through the cell cycle in the presence of genomic DNA damage in order to improve the efficiency of DNA repair (32). It has also been reported that $c-m y c$ directly regulates the transcription of the $n b s 1$ gene involved in DNA double-strand break repair (33). The present study showed that $c-m y c$ expression increased in irradiated NIH 3T3 cells with high expression of $m P e r 2$, thus suppressing cell death and enhancing cell repair.

BCL-2 and BAX are members of the BCL-2 family that is a key regulator of the mitochondrial response to apoptotic signals in the intrinsic pathway $(34,35)$. Bcl-2 is an antiapoptotic gene acting as a potent suppressor of apoptosis by blocking the release of cytochrome $c$, whereas bax is a proapoptotic gene with oppose functions acting as a promoter of cell death. The ratio of antiapoptotic-toproapoptotic molecules determines the response to a death signal (36). In the present study, the high expression of $m$ Per2 increased the BCL-2/BAX ratio, thus suppressing cell death and enhancing cell growth.

Wild-type P53 protein levels rise dramatically after exposure to ionizing radiation. This rise results from as yet undefined changes in the post-transcriptional modifications 
undergone by the P53 protein such as phosphorylation, binding to other proteins, or oligomerization. At subsequent end points of DNA-damage, a prolonged half-life was observed as well as increased DNA-binding activity of the $\mathrm{P} 53$ protein and enhanced transcriptional transactivation activity driven by this protein $(37,38)$. P53 can keep genomic stability by mediating apoptosis and DNA repair $(7-9,39)$ and can induce a transient arrest of the cell cycle at $\mathrm{G1}$, so that the cells will have time to repair damaged DNA. Activated p53 can also eliminate cells through mechanisms involving prolonged arrest at G1 or apoptosis. Our study showed an increased expression of p53 in irradiated NIH 3T3 cells at peak mPer2 expression, with decreased cell death. This indicated that a high expression of mPER2 up-regulated P53 expression, which could enhance DNA repair.

PCNA is a protein that acts as a processivity factor for DNA polymerase delta in eukaryotic cells. PCNA is an essential factor in cell proliferation and can be used as an index to evaluate cell proliferation (40). Since DNA polymerase delta is involved in resynthesis of excised damaged DNA strands during DNA repair, PCNA is important for both DNA

\section{References}

1. Lorimore SA, Wright EG. Radiation-induced genomic instability and bystander effects: related inflammatory-type responses to radiation-induced stress and injury? A review. Int J Radiat Biol 2003; 79: 15-25.

2. Cadet J, Bellon S, Douki T, Frelon S, Gasparutto D, Muller E, et al. Radiation-induced DNA damage: formation, measurement, and biochemical features. J Environ Pathol Toxicol Oncol 2004; 23: 33-43.

3. Bourguignon MH, Gisone PA, Perez MR, Michelin S, Dubner $D$, Giorgio MD, et al. Genetic and epigenetic features in radiation sensitivity. Part I: cell signalling in radiation response. Eur J Nucl Med Mol Imaging 2005; 32: 229-246.

4. Kent CR, Eady JJ, Ross GM, Steel GG. The comet moment as a measure of DNA damage in the comet assay. Int $J$ Radiat Biol 1995; 67: 655-660.

5. Willers H, Dahm-Daphi J, Powell SN. Repair of radiation damage to DNA. Br J Cancer 2004; 90: 1297-1301.

6. Wilson GD. Radiation and the cell cycle, revisited. Cancer Metastasis Rev 2004; 23: 209-225.

7. Biard DS, Martin M, Rhun YL, Duthu A, Lefaix JL, May E, et al. Concomitant p53 gene mutation and increased radiosensitivity in rat lung embryo epithelial cells during neoplastic development. Cancer Res 1994; 54: 3361-3364.

8. Kastan MB, Onyekwere O, Sidransky D, Vogelstein B, Craig RW. Participation of p53 protein in the cellular response to DNA damage. Cancer Res 1991; 51: 6304-6311.

9. Vogelstein B, Lane D, Levine AJ. Surfing the p53 network. Nature 2000; 408: 307-310.

10. Albrecht U, Eichele G. The mammalian circadian clock. Curr Opin Genet Dev 2003; 13: 271-277.

11. Pittendrigh CS. Temporal organization: reflections of a Darwinian clock-watcher. Annu Rev Physiol 1993; 55: 16-54.

12. Richter HG, Torres-Farfan C, Rojas-Garcia PP, Campino C, Torrealba F, Seron-Ferre M. The circadian timing system: synthesis and DNA repair. PCNA is also involved in the DNA damage tolerance pathway known as post-replication repair. In the present study, the irradiated cells with high expression of $m P e r 2$ showed enhanced cell proliferation, and the increase in PCNA staining after DNA damage could be a result of DNA repair.

After radiation, the high expression of mPer2 in $\mathrm{NIH}$ 3T3 cells results in reduced cell death and enhanced cell proliferation and clonogenic survival, which means that high expression of the circadian gene mPer2 diminishes radiosensitivity of $\mathrm{NIH} 3 \mathrm{~T} 3$ cells. A high expression of $m P e r 2$ may up-regulate the expression of apoptosis-related genes, with an increased proportionality of $b c /-2 / b a x$, and may reduce cell death and enhance cell proliferation to diminish radiosensitivity. Moreover, $m$ Per2 may also up-regulate DNA repair-related genes to increase DNA-repair efficiency after radiation to diminish radiosensitivity. Future research should be focused on studying the detailed mechanisms by which the circadian clock controls genes related to radiosensitivity.

making sense of day/night gene expression. Biol Res 2004; 37: 11-28.

13. Andretic R, Chaney S, Hirsh J. Requirement of circadian genes for cocaine sensitization in Drosophila. Science 1999; 285: 1066-1068.

14. Filipski E, King VM, Li X, Granda TG, Mormont MC, Liu X, et al. Host circadian clock as a control point in tumor progression. J Natl Cancer Inst 2002; 94: 690-697.

15. Haus E. Chronobiology of the mammalian response to ionizing radiation. Potential applications in oncology. Chronobiol Int 2002; 19: 77-100.

16. Fu L, Pelicano H, Liu J, Huang P, Lee $C$. The circadian gene Period2 plays an important role in tumor suppression and DNA damage response in vivo. Cell 2002; 111: 41-50.

17. Zhang J, Zhu B, Liu Y, Jiang Z, Wang Y, Li Y, et al. High expression of circadian gene mPer2 diminishes radiosensitivity of tumor cells. Cancer Biother Radiopharm 2008; 23 : 561-570.

18. Akashi M, Nishida E. Involvement of the MAP kinase cascade in resetting of the mammalian circadian clock. Genes Dev 2000; 14: 645-649.

19. Andretic R, Hirsh J. Circadian modulation of dopamine receptor responsiveness in Drosophila melanogaster. Proc Natl Acad Sci U S A 2000; 97: 1873-1878.

20. Dunlap JC. Molecular bases for circadian clocks. Cell 1999; 96: 271-290.

21. Reppert SM, Weaver DR. Molecular analysis of mammalian circadian rhythms. Annu Rev Physiol 2001; 63: 647-676.

22. Balsalobre A, Damiola F, Schibler U. A serum shock induces circadian gene expression in mammalian tissue culture cells. Cell 1998; 93: 929-937.

23. Yagita $\mathrm{K}$, Okamura $\mathrm{H}$. Forskolin induces circadian gene expression of rPer1, rPer2 and $\mathrm{dbp}$ in mammalian rat-1 fibroblasts. FEBS Lett 2000; 465: 79-82. 
24. Hua H, Wang Y, Wan C, Liu Y, Zhu B, Yang C, et al. Circadian gene mPer2 overexpression induces cancer cell apoptosis. Cancer Sci 2006; 97: 589-596.

25. Li L, Story M, Legerski RJ. Cellular responses to ionizing radiation damage. Int J Radiat Oncol Biol Phys 2001; 49: 1157-1162.

26. Khanna KK, Jackson SP. DNA double-strand breaks: signaling, repair and the cancer connection. Nat Genet 2001; 27: 247-254.

27. Dolganov GM, Maser RS, Novikov A, Tosto L, Chong S, Bressan DA, et al. Human Rad50 is physically associated with human Mre11: identification of a conserved multiprotein complex implicated in recombinational DNA repair. Mol Cell Biol 1996; 16: 4832-4841.

28. D'Amours D, Jackson SP. The Mre11 complex: at the crossroads of DNA repair and checkpoint signalling. Nat Rev Mol Cell Biol 2002; 3: 317-327.

29. Kobayashi J, Antoccia A, Tauchi H, Matsuura S, Komatsu K. NBS1 and its functional role in the DNA damage response. DNA Repair 2004; 3: 855-861.

30. Blackwell TK, Huang J, Ma A, Kretzner L, Alt FW, Eisenman $\mathrm{RN}$, et al. Binding of myc proteins to canonical and noncanonical DNA sequences. Mol Cell Biol 1993; 13: 52165224.

31. Evan GI, Vousden KH. Proliferation, cell cycle and apoptosis in cancer. Nature 2001; 411: 342-348.

32. Bil'din VN, Seregina TB, Pospelova TV. [The regulation of
DNA repair processes in mammalian cells. II. The repair of DNA radiation damage in NIH 3 T3 murine cells transformed by the v-myc oncogene]. Tsitologiia 1991; 33: 39-47.

33. Chiang YC, Teng SC, Su YN, Hsieh FJ, Wu KJ. c-Myc directly regulates the transcription of the NBS1 gene involved in DNA double-strand break repair. J Biol Chem 2003; 278: 19286-19291.

34. Adams JM, Cory S. The Bcl-2 protein family: arbiters of cell survival. Science 1998; 281: 1322-1326.

35. Cory S, Adams JM. The Bcl2 family: regulators of the cellular life-or-death switch. Nat Rev Cancer 2002; 2: 647-656.

36. Tsujimoto $\mathrm{Y}$. Cell death regulation by the $\mathrm{Bcl}-2$ protein family in the mitochondria. J Cell Physiol 2003; 195: 158-167.

37. Fei P, El-Deiry WS. P53 and radiation responses. Oncogene 2003; 22: 5774-5783.

38. Li CY, Nagasawa H, Dahlberg WK, Little JB. Diminished capacity for p53 in mediating a radiation-induced G1 arrest in established human tumor cell lines. Oncogene 1995; 11: 1885-1892.

39. Benchimol S. p53-dependent pathways of apoptosis. Cell Death Differ 2001; 8: 1049-1051.

40. Takasaki Y, Kaneda K, Takeuchi K, Matsudaira R, Matsishita $\mathrm{M}$, Yamada $\mathrm{H}$, et al. Analysis of the structure of proteasomeproliferating cell nuclear antigen (PCNA) multiprotein complex and its autoimmune response in lupus patients. Ann NY Acad Sci 2003; 987: 316. 